INPLASY

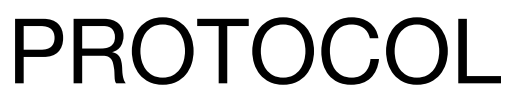

To cite: Ibeneme et al.

Effectiveness of mobile text reminder in improving quality of life, adherence to medication, and physical exercise in patients living with HIV: a protocol for systematic review. Inplasy protocol 202060016. doi: 10.37766/inplasy2020.6.0016

Received: 03 June 2020

Published: 03 June 2020

Corresponding author: Sandara Ndukwu

sophiachinenye95@gmail.com

Author Affiliation:

University of Nigeria, Enugu Campus

Support: This study received no funding.

Review Stage at time of this submission: Preliminary searches.

Conflicts of interest:

The authors declare that there is no conflict of interest.

\section{Effectiveness of mobile text reminder in improving quality of life, adherence to medication, and physical exercise in patients living with HIV: a protocol for systematic review}

Ibeneme, SC1; Ndukwu, SC2 ${ }^{2}$, Myezwa, $\mathrm{H}^{3}$; Irem, $\mathrm{FO}^{4}$; Ezenwankwo, EF5; Ajidahun, T6; Fortwengel, G.

Review question / Objective: adherence to HAART medication and physical exercise prescription in People living with HIV/ AIDS (PLWHA) based on reports from studies published in the databases from 1990 to August 2019?

Condition being studied: Human immunodeficiency syndrome (HIV) managed with highly active antiretroviral therapy (HAART).

Information sources: The bibliographic database and grey literature will be searched including eight bibliographic databases, namely - Allied and Complementary Medicine Database (AMED), Cumulative Index to Nursing and Allied Health Literature (CINAHL), Cochrane Library, EMBASE, EMCARE, Ovid MEDLINE, PsycINFO, and PubMed), trial registers and directory of open-access repository websites

INPLASY registration number: This protocol was registered with the International Platform of Registered Systematic Review and Meta-Analysis Protocols (INPLASY) on 03 June 2020 and was last updated on 03 June 2020 (registration number INPLASY202060016).

\section{INTRODUCTION}

Review question / Objective: Is mobile text reminder effective in improving quality of life (QoL), adherence to HAART medication and physical exercise prescription in People living with HIV/AIDS (PLWHA) based on reports from studies published in the databases from 1990 to August 2019?
Rationale: Mobile text reminder(SMS) is considered a viable strategy for targeting/ facilitating healthy behavioural change such as improved adherence to prescribed physical exercises (PE) and medication(antiretroviral therapy-ART) and likewise the quality of life(Qol) in people living with HIV/AIDS(PLWHA). Thus, the 
literature was appraised for evidence of its effectiveness.

Condition being studied: Human immunodeficiency syndrome (HIV) managed with highly active antiretroviral therapy (HAART).

\section{METHODS}

Search strategy: ((cellphone"OR"cellular phone"OR"reminder systems"OR"text messaging"OR"transportable cellular phone"OR"cellular telephone"OR"portable cellular phone" OR"mobile phones"OR"cell phone use "OR" reminder" OR"system "OR" messaging "OR" text" OR " short message service"OR"text message"OR" message "AND"hiv infections" OR"hiv-1"OR"hiv-2"OR"humans"OR"adult" OR"adolescent"OR"aged"OR"human immunodeficiency syndrome"OR"hiv longterm survivors"OR"seropositive individuals "AND"quality of life"OR"medication adherence" OR" treatment adherence and compliance" OR"patient compliance" OR"exercise" OR"physical activity"OR"exercise adherence"OR"physical activity adherenc)).

Participant or population: People living with HIV/AIDS (PLWHA).

Intervention: Mobile text reminder.

Comparator: Other treatment options including usual care or no treatment.

Study designs to be included: Randomized control trials (RCTs).

Eligibility criteria: Only RCTs will be involved, ii. studies that included mobile text reminders, iii. with outcomes that included quality of life, adherence to HAART and physical exercises, , iv. studies published in English, v. participants will be $\geq 18$ years.

Information sources: The bibliographic database and grey literature will be searched including eight bibliographic databases, namely - Allied and Complementary Medicine Database
(AMED), Cumulative Index to Nursing and Allied Health Literature (CINAHL), Cochrane Library, EMBASE, EMCARE, Ovid MEDLINE, PsycINFO, and PubMed), trial registers and directory of open-access repository websites.

Main outcome(s): The primary outcomes of interest will include adherence to drug prescription and physical activity. Studies that also investigated intervention effectiveness or quality of life will be included in this review.

Additional outcome(s): Studies will be included irrespective of the type of outcome measure utilized, provided any of the outcomes of interest were accessed.

Data management: Bibliographic records will be exported from RefWorks ${ }^{\mathrm{TM}}$ into Microsoft Excel (Microsoft 2013), to facilitate the management selection of articles for inclusion. The screening will be performed in two phases: the first phase will involve the screening of titles and abstracts utilizing the inclusion and exclusion criteria to identify potentially important articles and will be carried out by reviewer 1 . The results of the first screening will be independently crosschecked by reviewer 2 based on the review eligibility criteria. The second phase of screening will involve a screening of the articles selected from phase one screening by reviewer 1 . This will be cross-checked by reviewer 2. Differences of opinions occurring at any stage regarding inclusion or exclusion will be resolved by discussion and reflection, in consultation with reviewer 3. The reasons for excluding studies will be properly documented in the QUOROM flow chart.

Quality assessment / Risk of bias analysis: The methodological rigour of the selected studies will be assessed using the Physiotherapy Evidence Database (PEDro) quality appraisal tool. The PEDro is an 11item scale in which the first item relates to external validity and the other ten items assess the internal validity of a clinical trial. One point is given for each satisfying criterion (except for the first item) yielding 
a maximum score of 10 . The higher the score, the better the quality of the study and the following point scale will be used: 6-10 (High); 4-5 (fair or moderate); $\leq 3$ (poor). A point for a particular criterion will be awarded only if the article explicitly reported that the criterion was met. A score of one will be given for each yes answer and zero for no, unclear and not applicable (N/A) answers. The overall score will be reported as a tally of all "yes" answers out of 10 based on the applicable answers for each study. Scores of individual items from the critical appraisal tool will be added to present the total score.

Strategy of data synthesis: The review question on the effectiveness of mobile text reminders in enhancing the quality of life, adherence to HAART medication and physical exercise prescription in PLWHA will be answered. In doing this, all quantitative study outcomes which analyzed the effectiveness of this intervention will be presented, examined and combined in a proof table (Table 4). The proper statistical method will be used for different variables: for a continuous variable, weighted mean differences will be applied when outcomes are uniform or standard mean difference when different outcomes are used with $\mathrm{Cl}$ of $95 \%$ while for a dichotomous variable, the Risk ratio will be applied with $\mathrm{Cl}$ of $95 \%$. A meta-analysis will be done to find pooled effect sizes across studies, using a random-effect model unless there is a high level of heterogeneity of intervention effects. In which case, there will be a narrative synthesis. Heterogeneity will be assessed through the Cochran's $\times 2$ test $(10 \%$ significance level) and Higgins 12 for which values of $25 \%, 50 \%$ and $75 \%$ show low, medium and high heterogeneity respectively (as stipulated by the guidance in/the Cochrane Handbook for Systemic Reviews of Interventions).

Subgroup analysis: Sub-component analysis will be done to determine the effects of the intervention on all domains of the outcome measures, where applicable.
Sensibility analysis: Sensitivity analysis will be done to decide the impacts of studies with a high risk of bias on the general outcomes with and without these studies.

Language: Only studies published in English language will be included in this review.

Country(ies) involved: This study will be carried out in Nigeria.

Keywords: HIV, antiretroviral therapy, cell phone, text-message, medication Adherence, physical exercise adherence, Quality of life.

Dissemination plans: Findings of this study will be presented at clinical meetings, local and international scientific conferences and will be published in peer-reviewed journals.

\section{Contributions of each author:}

Author 1 - Sam lbeneme - SCI conceived the study, will participate in literature search and review, data extraction, study design and coordination, perform the statistical analysis, and help draft the manuscript.

Author 2 - Sandra Ndukwu - SN conceived the study, will participate in literature search and review, data extraction, study design and coordination, perform the statistical analysis, and help draft the manuscript.

Author 3 - Hellen Myezwa - Will participate in the design of the study, coordination, and help draft the manuscript.

Author 4 - Franklin onyedinma - Will Participate in literature search and review, data extraction and help draft the manuscript.

Author 5 - Elochukwu Ezenwankwo - Will participate in literature search and review, data extraction and help draft the manuscript.

Author 6 - Tunde Ajidahun - Will participate in the design of the study, coordination, and help draft the manuscript.

Author 7 - Gerhard Fortwengel - Will participate in data extraction and help draft the manuscript. 